\title{
A strategy for achieving low percolation and high electrical conductivity in melt-blended polycarbonate (PC)/multiwall carbon nanotube (MWCNT) nanocomposites: Electrical and thermo-mechanical properties
}

\author{
S. Maiti, N. K. Shrivastava, S. Suin, B. B. Khatua* \\ Materials Science Centre, Indian Institute of Technology, 721302 Kharagpur, India
}

Received 20 December 2012; accepted in revised form 16 February 2013

\begin{abstract}
In this work, polycarbonate (PC)/multiwall carbon nanotube (MWCNT) nanocomposites were prepared by simple melt mixing at a temperature $\left(\sim 350^{\circ} \mathrm{C}\right)$ well above the processing temperature of $\mathrm{PC}$, followed by compression molding, that exhibited percolation threshold as low as of $0.11 \mathrm{wt} \%$ and high electrical conductivity of $1.38 \times 10^{-3} \mathrm{~S} \cdot \mathrm{cm}^{-1}$ at only $0.5 \mathrm{wt} \%$ MWCNT loading. Due to the lower interfacial energy between MWCNT and PC, the carbon nanotubes are excellently dispersed and formed continuous conductive network structure throughout the host polymer. AC electrical conductivity and dielectric permittivity of PC/MWCNT nanocomposites were characterized in a broad frequency range, $10^{1}-10^{7} \mathrm{~Hz}$. Low percolation threshold $\left(p_{\mathrm{c}}\right)$ of $0.11 \mathrm{wt} \%$ and the critical exponent $(t)$ of $\sim 3.38$ was resulted from scaling law equation. The linear plot of $\log \sigma_{\mathrm{DC}} v s . p^{-1 / 3}$ supported the presence of tunneling conduction among MWCNTs. The thermal property and storage modulus of PC were increased with the incorporation of little amount of MWCNTs. Transmission electron microscopy (TEM) and field emission scanning electron microscopy (FESEM) confirmed the homogeneous dispersion and distribution of MWCNTs throughout the matrix phase.
\end{abstract}

Keywords: nanocomposites, electrical conductivity, thermal properties, PC

\section{Introduction}

In the last two decades, the electrically conductive polymer nanocomposites materials based on conductive carbon nanofillers or nanoparticles have become the subject of intensive research $[1,2]$. In general, polymer nanocomposites consist of twophase systems containing polymers with high surface area of nanofillers or nanoparticles. The field of polymer nanocomposites has been greatly illustrated with the incorporation of carbon nanotubes (CNTs) to improve their electrical, mechanical and thermal properties. The electrical conductive properties of polymer nanocomposites reinforced with CNTs at low concentration make a great advantage in the different field of applications. The homogeneous dispersion and distribution of CNTs throughout the matrix polymer is the main key that can help to decrease the filler loading concentration and improve the electrical, mechanical and thermal properties of the host polymer. The insulating polymer matrix can be changed to conductive polymer with the incorporation of sufficient amount of CNTs into host polymer. In the matrix polymer, an infinite cluster of interconnected conductive network structure has been developed with the incorporation of CNTs throughout the matrix polymer which helps to increase the electrical, thermal and mechanical properties of the polymer nanocompos-

\footnotetext{
${ }^{*}$ Corresponding author, e-mail: khatuabb@matsc.iitkgp.ernet.in (C) BME-PT
} 
ites. The polymer nanocomposites can be used in different field of applications such as organic LEDS [3], fuel cell membranes, photovoltaic devices [4], chemical sensors, transistors [5], catalysts [6], bioluminescent probes, high-performance and actuators [7] due its light weight and better processability. However, due to van der Waals interaction and lack of interfacial interactions among the CNTs, they are agglomerated in the host polymer and comparatively more amounts of CNTs are required to make the electrical conductive path in the polymer nanocomposites. The electrical conductivity of the polymer nanocomposites strongly depends on the concentration of nanofiller. The electrical conductivity of the nanocomposites was greatly improved by several orders of magnitude at the percolation threshold of the nanofiller in the matrix polymer. The percolation threshold coincides with the formation of an infinite interconnected conductive continuous network path throughout the polymer phase which help to increase the electrical conductivity. The key challenge for preparation of polymer-CNT nanocomposites is that individual dispersion of CNTs throughout the host polymer matrix upon melt blending. Generally, it has been demonstrated that there are excellent ways to disperse CNTs into thermoplastic matrices by three different methods for the preparation of polymer nanocomposites, such as (i) in-situ polymerization of monomer in presence of nanofillers, (ii) solution blending of polymer in presence of nanofillers, and (iii) melt blending of polymer with nanofillers [8].

PC has high impact resistance, toughness, dimensional stability and good optical clarity, and is mostly used in many engineering applications. PC has been modified by blending with various polymers for different applications and compounding with different kinds of CNTs to enhance the electrical, mechanical and thermal properties of the PC [912]. For instance, Wu et al. [9] have observed electrical conductivity of $2 \times 10^{-8} \mathrm{~S} \cdot \mathrm{cm}^{-1}$ with $2 \mathrm{wt} \%$ loading of MWCNT in melt blended PC/MWCNT nanocomposites, prepared by diluting solution blended PC/carboxylic acid functionalized MWCNT (10 wt\%) mixture. Hornbostel et al. [10] reported the percolation threshold of melt blended $\mathrm{PC} /$ MWCNT nanocomposites in between 1.5 to $2.0 \mathrm{wt} \%$ loading of MWCNT. Sathpathy et al. [11] have studied the electrical conductivity of melt blended PC/MWCNT nanocomposites and electrical con- ductivity of $2 \times 10^{-8} \mathrm{~S} \cdot \mathrm{cm}^{-1}$ was achieved at $2 \mathrm{wt} \%$ loading of MWCNT, prepared by diluting a PC/ MWCNT (15 wt\%) mixture. Yoon et al. [12] have studied the electrical percolation of melt blended PC/MWCNT nanocomposites prepared by diluting a PC/MWCNT mixture containing $20 \mathrm{wt} \%$ loading of MWCNT. They have shown the percolation threshold at $\sim 3 \mathrm{wt} \%$ MWCNT loading in the PC/ MWCNT nanocomposites.

This paper reported the preparation of highly electrical conducive PC/MWCNT nanocomposites at very low loading of MWCNT using the conventional melt-mixing technique and analyses the morphological and thermal properties of nanocomposites. To achieve this, melt blending of PC with MWCNT was carried out at $\sim 350^{\circ} \mathrm{C}$ (well above the processing temperature of $\mathrm{PC}$ ) that resulted in uniform dispersion and distribution of the MWCNTs throughout the host polymer and largely improved the electrical property even at very low MWCNT loading. Melt-mixing at this high temperature reduced the melt viscosity of $\mathrm{PC}$ and thus, increases the wettability of the polymer with the CNT that facilitated better dispersion of MWCNTs throughout the matrix polymer. Thus, high electrical conductivity of the PC/MWCNT nanocomposites was achieved at very low loading of MWCNT with lower percolation threshold. Literature on $\mathrm{PC} / \mathrm{CNT}$ nanocomposites revealed the preparation of the melt-mixed nanocomposites at the processing temperature $\left(280^{\circ} \mathrm{C}\right)$ of PC. In all the reported articles on $\mathrm{PC} / \mathrm{CNT}$ nanocomposites, the percolation of CNT in the composites is relatively of higher value. Thus, we assumed that the very high melt-viscosity of the PC may be one of the many reasons for poor dispersion of the CNTs in the matrix, due to insufficient wettability of the molten PC to the CNTs. Thus, lowering the melt-viscosity of the PC during melt-mixing of PC and CNT may improve the wettability of the polymer with CNTs and hence, lead to better dispersion of the CNTs in the PC matrix. To achieve this, we prepared the PC/MWCNT nanocomposites through conventional melt-mixing at a high temperature $\left(350^{\circ} \mathrm{C}\right)$. The morphological, electrical and thermal properties of the $\mathrm{PC} /$ MWCNT nanocomposites were studied by transmission electron microscopy (TEM), field emission electron microscopy (FESEM), dielectric measurement analysis, dynamic mechanical analysis (DMA) and thermogravimetric (TGA) analysis study. 


\section{Experimental}

\subsection{Materials details}

General purpose, transparent PC (Lexan 143; density: $1.19 \mathrm{~g} / \mathrm{cc}$; MFI: $10.5 \mathrm{~g} / 10 \mathrm{~min}$ at $300^{\circ} \mathrm{C}$ and $1.2 \mathrm{~kg}$ load) pellets (average diameter $\approx 2.75 \mathrm{~mm}$ and length $\approx 3.35 \mathrm{~mm}$ ) were obtained from SABIC Innovative Plastics (formerly General Electric). MWCNT employed in this study was of industrial grade (NC 7000 series; average diameter of $9.5 \mathrm{~nm}$ and length $1.5 \mu \mathrm{m}$; surface area $250 \sim 300 \mathrm{~m}^{2} / \mathrm{g}$; $90 \%$ carbon purity), purchased from Nanocyl S.A., Belgium. The electrical conductivity of the MWCNT is in the order of around $10^{4} \mathrm{~S} \cdot \mathrm{cm}^{-1}$. The MWCNTs were used as received, without any purification or chemical modification.

\subsection{Preparation of the $\mathrm{PC} / \mathrm{MWCNT}$ nanocomposites}

PC/MWCNT nanocomposites with different MWCNT loading were prepared by simple melt blending. At first, pure PC and unmodified MWCNTs were air dried at $100^{\circ} \mathrm{C}$ for $12 \mathrm{~h}$ to remove the moisture. After that, PC/MWCNT nanocomposites with different MWCNT $(0.3,0.5,0.8,1.0$ and $2.0 \mathrm{wt} \%)$ loading were prepared by simple melt blending using internal mixer (Brabender Plasticorder, with chamber capacity of $20 \mathrm{cc}$ ) at $350^{\circ} \mathrm{C}$ and $60 \mathrm{rpm}$ for $15 \mathrm{~min}$. PC/MWCNT nanocomposites were also prepared by melt-mixing PC with MWCNT for $15 \mathrm{~min}$ at $280^{\circ} \mathrm{C}$ and $60 \mathrm{rpm}$ for comparative studies. All the melt-mixed PC/MWCNT nanocomposites with different MWCNTs loadings were compression molded at $270^{\circ} \mathrm{C}$ and $4 \mathrm{MPa}$ pressure for further characterizations.

\section{Characterizations}

\subsection{Electrical conductivity}

The DC conductivity measurements were done on the molded specimen bars of dimensions $30 \times 10 \times$ $3 \mathrm{~mm}^{3}$. The sample was fractured at two ends and the fractured surface was coated with silver (Ag) paste to ensure good contact of the sample surface with electrodes. The electrical conductivities of the conducting composite were measured with a fourprobe technique. The specimens were prepared under similar conditions to avoid the influence of the processing parameters on the electrical properties. We applied current and measured the voltage in the four probe method. In the present study, the applied current was in the region from $10^{-2}$ to $10^{-5} \mathrm{~A}$ and the voltage obtained was in the range of 1 to 0.1 Volt for different samples.

Minimum of five tests were performed for each specimen and the data was averaged. For the dc electrical conductivity measurements, electrodes were in contact with the surface of the specimens. Direct current (DC) was applied for the dc electrical conductivity measurements. A current source forces a constant current and an ammeter measures the amount of current (I) passing through the sample. A voltmeter simultaneously measures the voltage $(V)$. The distance between electrodes is $1.25 \mathrm{~mm}$.

The dc electrical conductivity of the samples was calculated with the help of following Equations (1) and (2):

Resistivity $(\rho,[\Omega \cdot \mathrm{cm}])=\frac{V w h A^{*}}{I d}$

Conductivity $\left(\sigma,\left[\mathrm{S} \cdot \mathrm{cm}^{-1}\right]\right)=\frac{1}{\rho}$

where $V$ is the measured voltage, $I$ is the applied current, $w$ is the width of the sample bar, $h$ represents height of the sample bar, $d$ stands for distance between two points and $A^{*}$ is the geometric correction factor. The value of $A^{*}$ is $\sim 1$.

The frequency dependent $\mathrm{AC}$ conductivity and dielectric permittivity of the composites (disc type sample with thickness $0.3 \mathrm{~cm}$ and area $1.88 \times 10^{-1} \mathrm{~cm}^{2}$ ) were obtained using a computer controlled precision impedance analyzer (Agilent 4294A) by applying an alternating electric field (amplitude 1.000 Volt) across the sample cell in the frequency region of $40 \mathrm{~Hz}$ to $10 \mathrm{MHz}$. A parallel plate configuration is used for all the electrical measurements. Molded disc type composites sample was coated with silver (Ag) paste to act as both side electrodes. After electrode application, the samples were heated at $150^{\circ} \mathrm{C}$ in air to impart better adhesion between the sample and contacts. A sample holder using Pt probe was used for all the electrical measurements.

The parameters like dielectric permittivity $\left(\varepsilon^{\prime}\right)$ and dielectric loss tangent $(\tan \delta)$ were obtained as a function of frequency. The AC conductivity $\left(\sigma_{\mathrm{ac}}\right)$ was calculated from the dielectric data using the Equation (3):

$\sigma_{\mathrm{ac}} \approx \omega \varepsilon_{0} \varepsilon^{\prime} \tan \delta$

where $\omega$ is equal to $2 \pi f$ ( $f$ is the frequency), and $\varepsilon_{0}$ is vacuum permittivity. The dielectric permittivity $\left(\varepsilon^{\prime}\right)$ was determined with the following Equation (4): 
$\varepsilon^{\prime} \approx \frac{C_{\mathrm{p}}}{C_{0}}$

where $C_{\mathrm{p}}$ is the observed capacitance of the sample (in parallel mode), and $C_{0}$ is the capacitance of the cell. The value of $C_{0}$ was calculated using the area $(A)$ and thickness $(d)$ of the sample, following the Equation (5):

$C_{0} \approx \frac{\varepsilon \cdot A}{d}$

\subsection{High resolution transmission electron microscope (HRTEM)}

The extent of dispersion of the MWCNTs in the PC matrix phase was studied by HRTEM (HRTEM, JEM-2100, JEOL, JAPAN), operated at an accelerating voltage of $200 \mathrm{kV}$. The PC/MWCNT nanocomposites were ultra-microtomed at cryogenic condition, with sample thickness of around 70 90 nm. Since, the MWCNTs have higher electron density; they appeared as black lines in the TEM images.

\subsection{Field emission scanning electron microscope (FESEM)}

The surface morphology of the PC/MWCNT nanocomposites was studied using FESEM (FE-SEM, Carl Zeiss-SUPRA ${ }^{\mathrm{TM}}$ 40), with an accelerating voltage of $5 \mathrm{kV}$. The injection molded samples were dipped into the liquid nitrogen chamber for $35 \sim 40 \mathrm{~s}$, and were fractured under liquid nitrogen atmosphere. The cryo-fractured surfaces of the injection molded samples were gold coated with a thin layer (approx $\sim 5 \mathrm{~nm}$ ) to avoid the electrical charging. This gold coated nanocomposite samples were scanned in the vacuum order of $10^{-4}$ to $10^{-6} \mathrm{~mm} \mathrm{Hg}$ and FESEM images were taken on the fractured surface of the samples.

\subsection{Dynamic mechanical analysis (DMA)}

Thermo-mechanical properties of the pure PC and its nanocomposites with different MWCNTs loading were characterized by DMA (DMA 2980 model, TA Instruments Inc., USA). The dynamic mechanical spectra of the composites were obtained in tension film mode at a constant vibration frequency of $1.0 \mathrm{~Hz}$, with a preload force of $10 \mathrm{mN}$, static stress/ dynamic stress amplitude ratio ('force tracking') of $110 \%$, temperature range of $30-180^{\circ} \mathrm{C}$ at a heating rate of $5^{\circ} \mathrm{C} / \mathrm{min}$, under $\mathrm{N}_{2}$ atmosphere. The dimension of the specimen was $30 \times 6.40 \times 0.42 \mathrm{~mm}^{3}$.

\subsection{Thermogravimetric analysis (TGA)}

The thermal stability of the pure $\mathrm{PC}$ and its nanocomposites with different MWCNTs loading were studied using TGA (TGA-209F, from NET$\mathrm{ZSCH}$, Germany; temperature accuracy: $\pm 0.5^{\circ} \mathrm{C}$ ). TGA was carried out from room temperature to $800^{\circ} \mathrm{C}$ under $\mathrm{N}_{2}$ atmosphere with a heating rate of $10^{\circ} \mathrm{C} / \mathrm{min}$. The degradation temperatures of the samples at various stages were calculated from the TGA curves.

\section{Results and discussion \\ 4.1. Electrical analysis \\ 4.1.1. DC conductivity}

Figure 1a shows the DC electrical conductivity of the melt-mixed PC/MWCNT nanocomposites prepared at $280^{\circ} \mathrm{C}$. At low CNT loading (below $1 \mathrm{wt} \%$ ), the nanocomposites resemble the conductivity value similar to that of the pure PC. It was noteworthy; a rapid increase ( 3 orders of magnitude) in conductivity of the nanocomposites was observed when the MWCNT loading was increased to $1.1 \mathrm{wt} \%$. Beyond this loading, electrical conductivity of the nanocomposites gradually increases with increasing content of MWCNT. Thus, electrical conductivity of $2.31 \times 10^{-9} \mathrm{~S} \cdot \mathrm{cm}^{-1}$ was achieved at high MWCNT loading $(1.6 \mathrm{wt} \%)$. The DC electrical conductivity of the nanocomposites was $5.12 \times 10^{-8} \mathrm{~S} \cdot \mathrm{cm}^{-1}$ at $2.0 \mathrm{wt} \%$ of MWCNT loading and $2.12 \times 10^{-6} \mathrm{~S} \cdot \mathrm{cm}^{-1}$ of electrical conductivity was achieved at very high MWCNT loading (3.0 wt \%), as shown in Figure 1a. Sathpathy et al. [11] have also studied the electrical conductivity of melt blended PC/MWCNT nanocomposites and reported the electrical conductivity value of $2 \times 10^{-8} \mathrm{~S} \cdot \mathrm{cm}^{-1}$ at $2 \mathrm{wt} \%$ MWCNT loading. From the plot, the percolation threshold $\left(p_{\mathrm{c}}\right)$ of MWCNT in the nanocomposites was calculated using Equation (6), as given below. A well fitted linear plot of $\log \sigma_{\mathrm{DC}} v s . \log \left(p-p_{\mathrm{c}}\right)$ was plotted (shown in inset Figure 1a) with the help of the Equation (6) to calculate the value of ' $p_{\mathrm{c}}$ ' for the PC/MWCNT nanocomposites and the pc was found at $\approx 1.1 \mathrm{wt} \%$ ( $\sim 0.98 \mathrm{vol} \%)$ MWCNT in the nanocomposites.

Thus, the $\sigma_{\mathrm{DC}}$ of the polymer nanocomposites as a function of $p_{\mathrm{c}}$ can be expressed by the following scaling law Equations (6) and (7): 
$\sigma_{\mathrm{DC}}(p)=\sigma_{0}\left(p-p_{\mathrm{c}}\right)^{\mathrm{t}}$ for $p>p_{\mathrm{c}}$

$\sigma_{\mathrm{DC}}(p)=\sigma_{0}\left(p_{\mathrm{c}}-p\right)^{\mathrm{t}}$ for $p<p_{\mathrm{c}}$
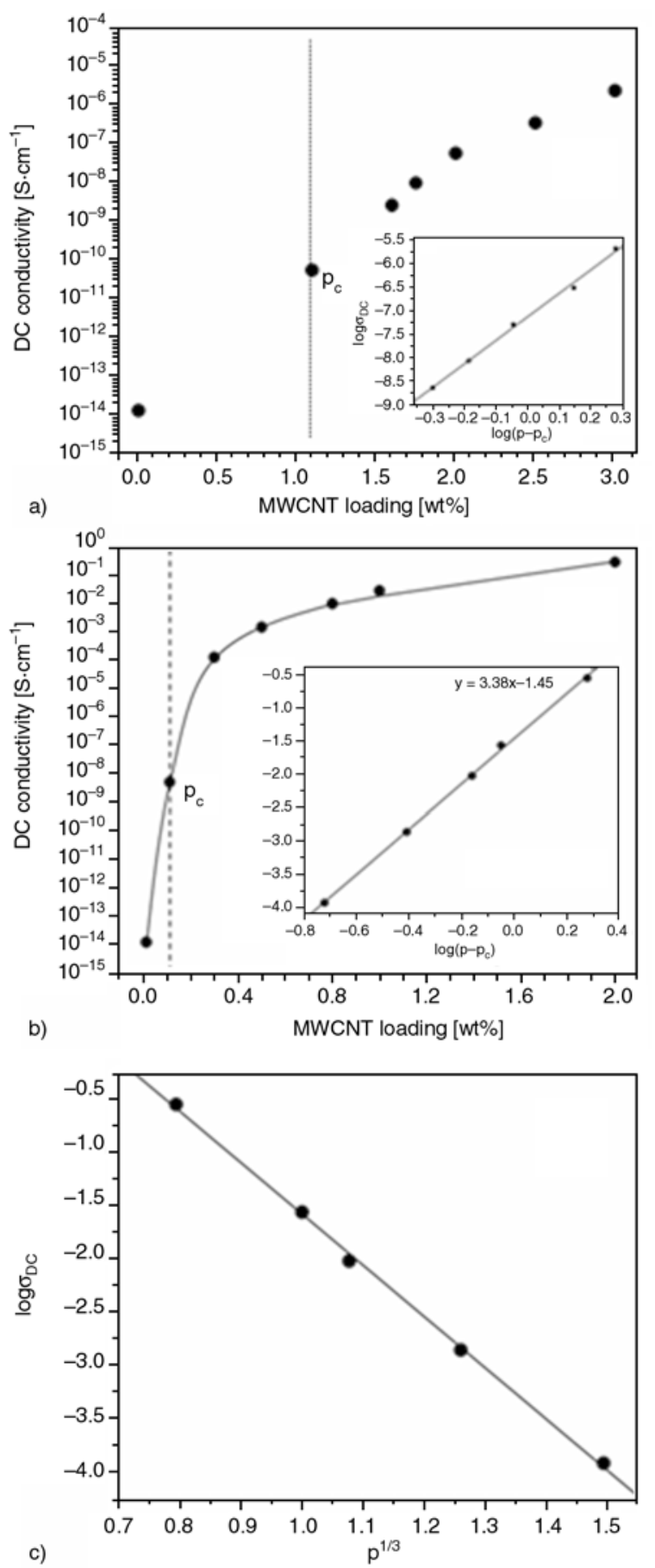

Figure 1. Plot of DC conductivity $v s$. MWCNT loading of PC/MWCNT nanocomposites prepared at (a) $280^{\circ} \mathrm{C}$; (b) $350^{\circ} \mathrm{C}$; and (c) $\log \sigma_{\mathrm{DC}} v s \cdot p^{-1 / 3}$ for $\mathrm{PC} / \mathrm{MWCNT}$ nanocomposites prepared at $350^{\circ} \mathrm{C}$. The inset in Figure 1a and $1 \mathrm{~b}$ represents the plot for $\log \sigma_{\mathrm{DC}} v s . \log \left(p-p_{\mathrm{c}}\right)$ for the nanocomposites. The straight line in the inset (Figure 1b) is a least-square fit to the data using Equation (6) giving the best fit values $p_{\mathrm{c}} \approx 0.11 \mathrm{wt} \%$ and $t \approx$ 3.38 . where $p_{\mathrm{c}}$ stands for the percolation threshold, depends on the lattice in which particles are accommodated and ' $s$ ' and ' $t$ ' symbolize for critical exponents. The ' $s$ ' and ' $t$ ' values depend on the dimensionality and the percolation threshold value of the polymer nanocomposites [13].

The DC electrical conductivity $\left(\sigma_{\mathrm{DC}}\right)$ of the $\mathrm{PC} /$ MWCNT nanocomposites, prepared at $350^{\circ} \mathrm{C}$, with different MWCNT loading is shown in Figure 1b. It is clearly observed from the figure that the $\sigma_{\mathrm{DC}}$ value of the PC/MWCNT nanocomposites gradually increased with increasing the wt $\%$ of MWCNT loading into the PC/MWCNT nanocomposites. The $\sigma_{\mathrm{DC}}$ value of $1.19 \times 10^{-4} \mathrm{~S} \cdot \mathrm{cm}^{-1}$ was achieved when PC/MWCNT nanocomposites was prepared with extremely low loading $(0.3 \mathrm{wt} \%)$ of MWCNT. This high electrical conductivity of the PC/MWCNT nanocomposites at very low loading of MWCNTs is the indication for the development of continuous conductive interconnected network structure of CNT-CNT throughout the matrix polymer. This is the reason for increasing the net $\sigma_{\mathrm{DC}}$ of the $\mathrm{PC} /$ MWCNT nanocomposites with increasing the MWCNT loading in the nanocomposites. We assume that the obtained high conductivity values are due to good dispersion of the MWCNT in PC matrix as a result of relatively low melt-viscosity of the PC during melt-mixing at high temperature.

Initially, $\sigma_{\mathrm{DC}}$ value $\left(1.1 \times 10^{-14} \mathrm{~S} \cdot \mathrm{cm}^{-1}\right)$ of the $\mathrm{PC} /$ MWCNT nanocomposites with $0.01 \mathrm{wt} \%$ MWCNT loading was almost same with the electrical conductivity value of insulating pure PC. Thus, the electrical conductivity of the pure PC was not affected with the incorporation of very small amount $(0.01 \mathrm{wt} \%)$ of MWCNTs in the nanocomposites. However, when PC/MWCNT nanocomposites was prepared with $0.3 \mathrm{wt} \%$ loading of MWCNTs, the $\sigma_{\mathrm{DC}}$ value of the nanocomposites tremendously increased to $1.19 \times 10^{-4} \mathrm{~S} \cdot \mathrm{cm}^{-1}$. This rapid change in electrical conductivity of the nanocomposites by several orders (10 orders) of magnitude from $10^{-14}$ to $10^{-4}$ is the indication for the formation of continuous interconnected conductive network chains of CNT-CNT throughout the host polymer, which is well known as percolation network. Furthermore, the $\sigma_{\mathrm{DC}}$ of the PC/MWCNT nanocomposites again increased with increasing the MWCNTs loading in the nanocomposites, as shown in Figure 1b. Thus, the $\sigma_{\mathrm{DC}}$ of $1.38 \times 10^{-3} \mathrm{~S} \cdot \mathrm{cm}^{-1}$ was achieved at $0.5 \mathrm{wt} \%$ MWCNT loading, $9.46 \times 10^{-3} \mathrm{~S} \cdot \mathrm{cm}^{-1}$ at $0.8 \mathrm{wt} \%$ 
MWCNT loading and $2.73 \times 10^{-2} \mathrm{~S} \cdot \mathrm{cm}^{-1}$ at $1.0 \mathrm{wt} \%$ loading of MWCNT.

Basically, percolation theory $[7,14]$ explained the variation of $\sigma_{\mathrm{DC}}$ with the different loading $(p)$ of conducting nanofillers or nanoparticles in conducting polymer nanocomposites. The percolation theory can be defined both theoretically and experimentally. According to percolation theory, the transition occurred from an insulating materials to a conductor at a certain concentration of the conducting nanofillers, known as critical concentration, where the filler concentration are enough to form a continuous interconnected conductive network path through the insulating polymer phase. This minimum concentration of the conducting nanofillers at which electrical conductivity of the nanocomposites rapidly increased is known as the percolation threshold $\left(p_{\mathrm{c}}\right)$. A schematic representation for arrangement of MWCNTs in the PC/MWCNT nanocomposites at the percolation threshold is shown in Figure 2.

A well fitted linear plot of $\log \sigma_{\mathrm{DC}} v s . \log \left(p-p_{\mathrm{c}}\right)$ was plotted with the help of the Equation (6) to calculate the value of ' $t$ ' and ' $p_{\mathrm{c}}$ ' for the PC/MWCNT nanocomposites, as shown in the inset of Figure $1 \mathrm{~b}$. The calculated $p_{\mathrm{c}}$ and ' $t$ ' values for the PC/MWCNT nanocomposites was $(0.11 \mathrm{wt} \% \quad(\sim 0.097 \mathrm{vol} \%)$ MWCNT loading and $\sim 3.38$, respectively. This very low pc value $(0.11 \mathrm{wt} \%)$ suggested that $0.11 \mathrm{wt} \%$ MWCNT loading is sufficient for the formation of continuous conductive CNT-CNT network path throughout the host polymer. Thus, this kind of network path of MWCNTs throughout the matrix phase is the reason behind the high $\sigma_{\mathrm{DC}}$ of the $\mathrm{PC} /$ MWCNT nanocomposites.

Several researchers $[6,15]$ have studied the critical exponent $(t)$ values of different polymer nanocomposites systems. They have explained it on the basis of theoretical study especially for two dimensional (2D) and three dimensional (3D) lattices. The calculated ' $t$ ' value for $2 \mathrm{D}$ lattice is varied from 1.10 to
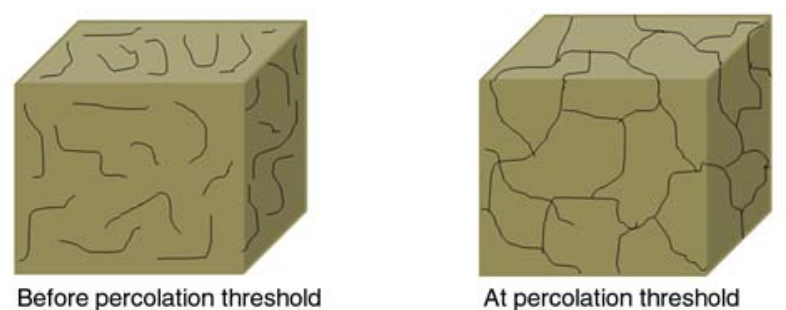

Figure 2. A schematic presentation for the arrangement of MWCNTs before percolation threshold and at percolation threshold in PC/MWCNT nanocomposites
1.43 and for 3D lattice system, obtained ' $t$ ' value is lower than 2.02. Several research groups $[16,17]$ have studied the electrical conductivity of different polymer/CNT nanocomposites and the estimated ' $t$ ' values are observed in the range from 1.3 3.9, depending on the matrix polymer and the type of CNT in the composites.

Balberg et al. [18] expressed that the percolation threshold is related with average excluded volume by the following Equation (8):

$p_{\mathrm{c}}\left(\frac{L}{R}\right) \approx 3$

where $p_{\mathrm{c}}$ represents the percolation threshold which is expressed as the partial volume of the nanofiller and $L$ is considered as the length of the randomly oriented stick particles and $R$ signifies the radius of the stick particles.

Grossiord et al. [19] have explained that the electrical conductivity in the polymer nanocomposites might be developed because of tunneling mechanism effect. Two particles are supposed to be connected when they are in physical contact. Due to the inter-particle conduction mechanism, polymer nanocomposites are showing higher electrical conductivity than that of the insulating polymer matrix before formation of continuous interconnected network path of nanofillers in the nanocomposites. Ryvkina et al. [20] expressed that electron tunneling mechanism dominated the conduction in the polymer/CB nanocomposites and explained it with the help of following theoretical model shown in Equation (9):

$\sigma_{\mathrm{DC}} \propto \exp (-A d)$

where $A$ signifies the tunnel parameter and $d$ stands for the tunnel distance, respectively. In recent times, tunneling conduction mechanism in the different nanocomposites systems has been well explained in the literature $[21,22]$. According to tunneling conduction mechanism, the charge carriers move through the polymer nanocomposites across insulating gaps between nanofillers and generated electrical conduction in the nanocomposites. The charge carriers can be developed by physical contact between the nanofillers or even they are separated by insulating gaps in their pathways. Thus, electrical conductivity of the polymer nanocomposites can be varied due to the existence of tunneling conduction which contributes to the current through the polymer 
nanocomposites [23]. The current in a tunnel junction exponentially decreases with the barrier width. If nanofillers are randomly distributed, then the mean average distance $(d)$ among the nanofillers would be barrier width. This mean average distance is considered, in the first approximation, to be directly proportional to the nanofillers concentration in weight, $p^{-1 / 3}$ and can be expressed by the following Equation (10) [24]:

$d \propto p^{-1 / 3}$

Thus, the combination of the Equations (9) and (10), shows that tunneling assisted conductivity $\left(\log \sigma_{\mathrm{DC}}\right)$ is directly proportional with $p^{-1 / 3}$ and expressed by the following Equation (11):

$\log \left(\sigma_{\mathrm{DC}}\right) \propto p^{-1 / 3}$

Figure $1 \mathrm{c}$ shows the linear variation of $\log \sigma_{\mathrm{DC}}$ with $p^{-1 / 3}$ for PC/MWCNT nanocomposites. This linear variation of $\log \sigma_{\mathrm{DC}}$ with $p^{-1 / 3}$ supported the existence of tunneling mechanism in the nanocomposites which is the one of the reasons behind the electrical conductivity. Kilbride et al. [25] expressed that the electrical contact between the nanofillers will be less and contact resistance among them will be high when individual nanofillers were coated by insulating polymer matrix in the polymer nanocomposites. The dispersion of MWCNTs during the preparation of the polymer nanocomposites is such that the 'arms' of the MWCNTs get entangled while their centers still form a random distribution. Thus, electrically conductive MWCNTs are separated by the insulating thin polymer in the nanocomposites [26, 27]. Therefore, tunneling of the electrons happened among the adjacent CNTs through very thin layer of insulating polymer matrix. Here, MWCNT acts as an electrode and cannot contact with each other exactly. They are always separated in a margin of $\mathrm{nm}$ apart. We are not able to predict the exact field intensity and exact number of conducting path as the cross section of MWCNTs is differing from CNT to CNT. Tunneling is also sensitive to the distance between the MWCNTs. Generally, a charge carrier cannot hop in insulating polymer from a conductive site to another adjacent one due to high potential energy barrier. This high energy barrier gap can be reduced by applying voltage from the source between the two electrodes (or conductive sites). Thus, electrons move freely between the electrodes by tunneling conduction mechanism when energy barrier is sufficiently less. Thus, contact resistance is reduced and the electrical conductivity of the polymer nanocomposites is increased. The property of the energy barrier depends on the matrix polymer and also the fabrication method. In the case of polymer nanocomposites with semicrystalline polymer, nanofiller may act as a nucleating agent on the amount of lamellae that may develop around the nanofiller [4].

\subsubsection{AC conductivity}

The AC electrical conductivity of the PC/MWCNT nanocomposites as a function of frequency $(f)$ is observed in Figure 3. This measurement is carried out in the broad band frequency range of $10^{1}$ to $10^{7} \mathrm{~Hz}$ at room temperature. As can be seen, $0.1 \mathrm{wt} \%$ $\mathrm{PC} / \mathrm{MWCNT}$ nanocomposite is showing slightly higher electrical conductivity than that of pure PC. However, the electrical conductivity of the $\mathrm{PC} /$ MWCNT (0.1 wt\%) nanocomposites gradually increased with frequency like pure PC. In the beginning, the electrical conductivity of the nanocomposites was increased drastically with increasing the loading of MWCNT in the nanocomposites and later, the rate of increase was comparatively less in case of higher loading, shown in Figure 3.

In the beginning, the $\mathrm{AC}$ electrical conductivity of the PC/MWCNT nanocomposites does not depend on frequency and shows almost same value up to a certain frequency.

Thus, AC electrical conductivity remains almost constant until a certain frequency, known as critical frequency $\left(f_{\mathrm{c}}\right)$. This incident is clearly shown in Fig-

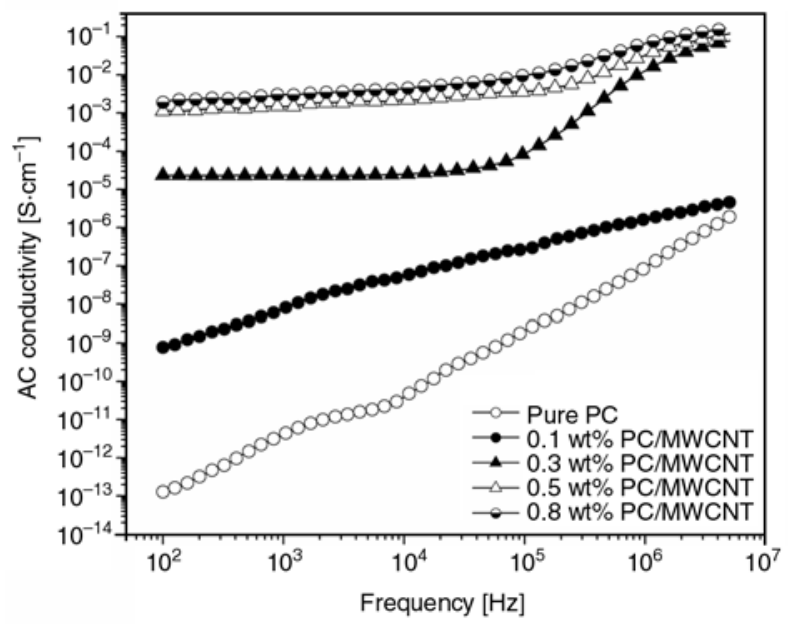

Figure 3. AC conductivity of the pure $\mathrm{PC}$ and $\mathrm{PC} / \mathrm{MWCNT}$ nanocomposites prepared at $350^{\circ} \mathrm{C}$ versus frequency at different MWCNT loadings 
ure 3. Thus, the AC electrical conductivity strongly depends on the frequency when the frequency $(f)$ is greater than the critical frequency $\left(f_{\mathrm{c}}\right)$ (i.e. $f>f_{\mathrm{c}}$ ). This kind of behavior of frequency dependent AC electrical conductivity, in the first approximation value, can be expressed by the following Equation (12).

$\sigma_{\mathrm{AC}} \propto f^{\mathrm{s}}$

where $s$ signifies as an exponent.

According to percolation theory, variation of AC electrical conductivity with the frequency is expected to be caused by the influence of large polymeric gaps among the conducting clusters. As finite-size clusters are self-similar fractals [28], the size of the finite-size cluster is increased with increasing the frequency. This is the reason for increasing electrical conductivity of the nanocomposites with the frequency. As nanofiller concentration increases, a continuous conductive network of physically connected nanotubes appears through the polymer [29]. AR von Hippel [30] discusses about ac conductivity in its bible of dielectrics 'Dielectrics and waves' and defines that ac conductivity is the summation of all dissipative effects including an actual ohmic conductivity. This is caused by migrating charge carriers as well as a frequency dielectric dispersion. Several research groups $[31,32]$ explained that the electron hopping and electron tunneling mechanism among the nanofillers is the reason for the development of AC electrical conductivity in the polymer nanocomposites. In the polymer nanocomposites, nanofillers form an interconnected conductive network structure throughout the host polymer and this will help to improve the rate of electron hopping or electron tunneling above a percolation threshold. Thus, AC electrical conductivity in the nanocomposites is increased and it is directly proportional with the electron hopping and also electron tunneling. With the increasing of nanofillers concentration in the nanocomposites, development of the interconnected conductive network structure increased and significantly increased the electrical conductivity of the nanocomposites.

The AC electrical conductivity $\left(\sigma_{\mathrm{AC}}\right)$ of any dielectric material below $f_{\mathrm{c}}$ (at low frequency range) is the summation of two components and can be expressed in terms of DC electrical conductivity $\left(\sigma_{\mathrm{DC}}\right)$, angular frequency ( $\omega$, which is equal to $2 \pi f)$ and dielectric loss factor $\left(\varepsilon^{\prime \prime}\right)$ by the following Equation (13):
$\sigma_{\mathrm{AC}}=\sigma_{\mathrm{DC}}+\omega \varepsilon^{\prime \prime}$

The first component of the equation is the $\sigma_{\mathrm{DC}}$ which arises due to the ionic or electronic conductivity and the value of the second component $\left(\omega \varepsilon^{\prime \prime}\right)$ in the relation strongly depends on the extent of polarization of dipoles (permanent and induced) and accumulated interfacial charges, known as MaxwellWagner-Sillars (MWS) effect [33, 34]. Polarization effects based on different physical origins appear at various frequency regions. Electrode polarization and/or interfacial polarization occur at low frequency regions. At relatively higher frequencies glass to rubber transition process is observed, and at even higher frequencies weak phenomena resulting from local motions of small parts of the polymer are present. The frequency where a relaxation process is recorded is related to the nature of the specific dipole and to their inertia to the field's alternation (relaxation time). At low frequency region, more and more charge carriers accumulate at the interface between the samples and electrode, which help to drop in ac conductivity at low frequency region [35]. The effect of interfacial polarization becomes more significant at low frequency region (below $f_{\mathrm{c}}$ ) as the dipoles/induced dipoles get enough time to orient themselves with the direction of applied electric field (relaxation phenomena). Thus, the value of $\sigma_{\mathrm{AC}}$ for a conductive system truly represents the $\sigma_{\mathrm{DC}}$ at low frequency region. The frequency independence of electrical conductivity for different disordered materials below critical frequency $\left(f_{\mathrm{c}}\right)$ has already been reported [36, 37].

At high frequency region (above $f_{\mathrm{c}}$ ), the polarization effect becomes unimportant as the dipoles get less relaxation time to orient themselves in the direction of applied electric field. The applied AC electric field (periodic alternation) above $f_{\mathrm{c}}$ results in the radical reduction of space charge accumulation and orientation of dipoles in the applied field direction that reduce in value of polarization. Thus, the value of $\sigma_{\mathrm{AC}}$ strongly depends on the excitation of the charge particles and flow of electrons through the continuous conductive network in the matrix phase. At high frequency region, localized charge carriers motion makes it possible to take maximum advantage for high conductivity. While at low frequency region, charge transport must extend over longer distances and resulted low conductivity [31]. Conductivity increases rapidly with frequency, after 
a critical value, because charge carriers migrate to shorter distances as frequency is raised due to the alternation of the field. Adjacent conductive sites facilitate carriers to move back and forward overcoming low energy barriers, enabling thus higher values of conductivity.

\subsubsection{Dielectric permittivity}

The dielectric permittivity $\left(\varepsilon^{\prime}\right)$ of the pure PC and $\mathrm{PC} / \mathrm{MWCNT}$ nanocomposites as a function of frequency was measured in the broad band frequency range $50 \sim 10^{7} \mathrm{~Hz}$ at room temperature. The variation of dielectric permittivity of the pure $\mathrm{PC}$ and $\mathrm{PC} /$ MWCNT nanocomposites with frequency is shown in Figure 4a.

Initially, the dielectric permittivity of neat $\mathrm{PC}$ has a low value, which decreased marginally with increasing frequency. The dielectric permittivity of the PC/MWCNT nanocomposites was high and then slowly decreased with increase in the frequency at lower frequency range. This is the well known behavior of any dielectric materials [38]. The polarization effect is the reason for the variation of dielectric permittivity with the frequency at low frequency region. The dielectric permittivity of the materials strongly depends on the polarizability [39]. At low frequency region, the polarization effect is more significant and molecules of dielectric materials get enough relaxation time to orient them in the direction of the applied electric field when voltage is applied. This is the reason for high dielectric permittivity of the nanocomposites at low frequency region. However, polarization effect become insignificant at high frequency region because of molecules is not getting sufficient relaxation time for orientation themselves in the direction of applied alternative electric field. Thus, dielectric permittivity is sharply reduced in the nanocomposites after a certain frequency, as shown in Figure 4a.

The dielectric permittivity of the nanocomposites not only depends on the frequency but also on the nanofiller concentrations. As observed, dielectric permittivity is increased with increasing the concentration of MWCNTs in the nanocomposites at constant frequency $\left(\sim 10^{4} \mathrm{~Hz}\right)$. Figure $4 \mathrm{~b}$ represents the plot dielectric permittivity $\left(\varepsilon^{\prime}\right)$ vs. MWCNT loading, at constant frequency. This Figure $4 \mathrm{~b}$ concluded that the value of $\varepsilon^{\prime}$ increases with increasing the MWCNT loading in the nanocomposites at constant frequency $\left(\sim 10^{4} \mathrm{~Hz}\right)$. The incorporation of
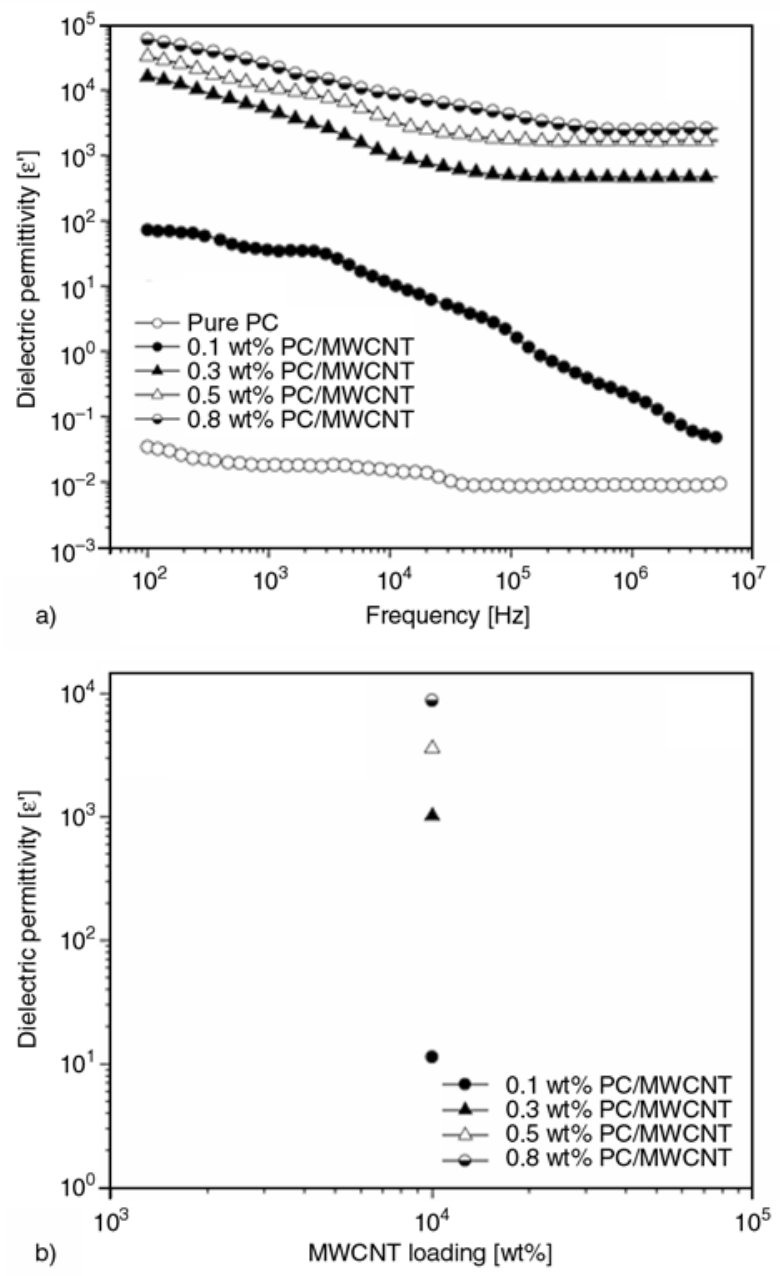

Figure 4. Dielectric Permittivity vs. frequency of the pure $\mathrm{PC}$ and PC/MWCNT nanocomposites with (a) different MWCNT loading and (b) dielectric permittivity $v s$. MWCNT loading at constant frequency of the PC/MWCNT nanocomposites, prepared at $350^{\circ} \mathrm{C}$ at different MWCNT loadings

nanofillers in the nanocomposites may increase the dielectric permittivity due to several factors, such as (i) the morphology of the polymer in the nanocomposites is changed in the presence of nanofillers, (ii) the large surface area and nano-sized nanofillers creates a large interaction zone with the neighbors in the polymer nanocomposites [2], (iii) the space charge distribution in the nanocomposites has been changed for the high electrical conductive nature of nano-sized nanofillers [8], and (iv) also changes the scattering mechanism of the nanocomposites. Dissado and Hill [40] explained the dielectric permittivity of the nanocomposites and stated that the value of the dielectric permittivity is high at low frequency region due to 'quasi-dc' conduction. Lewis [41] explained 'quasi-dc' conduction with the help of O'Konski's model [42] and a double 
layer approach. According to the O'Konski's model, the charge carriers are efficiently moved around the interface by the field leading to an induced polarization at the polar ends of the fillers or particles [41]. In the case of nanocomposites materials reinforced with nanofillers, the nanofillers leads to a remarkably large interfacial area in the nanocomposites due to its high surface area. Thus, nanofillers hold a much greater interface with their surroundings rather than macrofillers due to its nano-sized and high aspect ratio and the degree of interfacial interaction between the nanofiller and the matrix polymer has been demonstrated by this interface.

\subsection{Morphology study}

The scanning electron micrographs of PC/MWCNT nanocomposites are shown in Figure 5. Figure 5a represents the FESEM image of the PC/MWCNT nanocomposites, which indicated the formation of a continuous interconnected conductive network structure of the MWCNTs throughout the polymer phase in the nanocomposites. These micrographs also indicated that MWCNTs were homogeneously and regularly distributed throughout the matrix phase along with minor agglomeration. We assume that the extent of distribution and dispersion of the MWCNTs at $0.3 \mathrm{wt} \%$ loading was sufficient to develop the continuous interconnected conductive network structure in the nanocomposites which resulted in the electrical conductivity. From the TEM image (Figure $5 b$ ), random and homogeneous dispersion of rod like structures clearly indicated the MWCNTs with retention of their aspect ratio (without much breaking or damage of nanotubes) in matrix phase of the nanocomposites prepared by melt-blending followed by compression molding.

\subsection{Thermal analysis \\ 4.3.1. TGA}

The thermal stability of the host polymer is significantly increased with the addition of MWCNTs because of its restricted chain mobility of the polymer near the nanofillers surface. During combustion, MWCNTs act as inflammable anisotropic nanoparticles forming a jammed network of char layers that retards transport of the decomposition products. Thermal stability of the pure $\mathrm{PC}$ and $\mathrm{PC} /$ MWCNT nanocomposites, prepared at $350^{\circ} \mathrm{C}$, was characterized by the TGA thermogram under $\mathrm{N}_{2}$ atmosphere, as shown in Figure 6. From the figure, it is clearly seen that the thermal decomposition temperature and the residual mass of the PC/MWCNT nanocomposites increased when MWCNT was incorporated into the PC matrix. The initial degradation temperature $\left(T_{1}\right.$; the temperature corresponds to $10 \%$ weight loss) and $50 \%$ degradation temperature $\left(T_{50}\right)$ of the pure PC and its nanocomposites with different MWCNT loading were calculated.

The pure PC shows its initial degradation $\left(T_{1}\right)$ temperature at $\left(475^{\circ} \mathrm{C}\right.$ and $T_{50}$ at $\sim 510^{\circ} \mathrm{C}$. The calculated values of $T_{1}$ and $T_{50}$ for the PC/MWCNT nanocomposites containing $0.3 \mathrm{wt} \%$ MWCNT loading were $\left(502^{\circ} \mathrm{C}\right.$ and $\sim 547^{\circ} \mathrm{C}$, respectively. Thus,

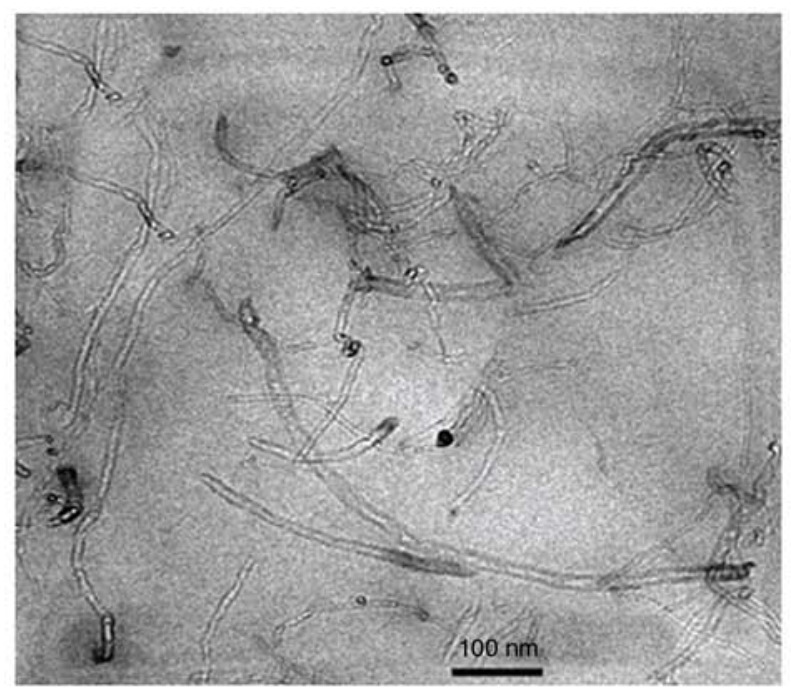

b)

Figure 5. FESEM micrograph (a), and TEM micrograph (b) of PC/MWCNT nanocomposites, prepared at $350^{\circ} \mathrm{C}$, containing $0.3 \mathrm{wt} \%$ of MWCNT 


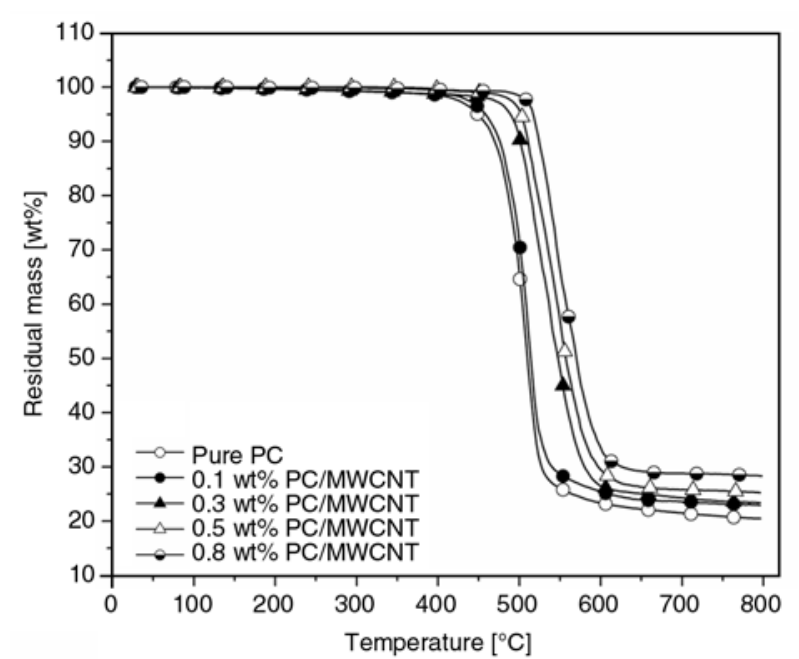

Figure 6. TGA thermograms of pure $\mathrm{PC}$, and $\mathrm{PC} / \mathrm{MWCNT}$ nanocomposites containing $0.1 \mathrm{wt} \% \mathrm{MWCNT}$ loading, $0.3 \mathrm{wt} \%$ MWCNT loading, $0.5 \mathrm{wt} \%$ MWCNT loading, $0.8 \mathrm{wt} \%$ MWCNT loading. All the nanocomposites were prepared at $350^{\circ} \mathrm{C}$.

thermal stability of the PC/MWCNT naocomposites with $0.3 \mathrm{wt} \%$ MWCNT loading was superior to the pure PC. MWCNT acts as a protective barrier against thermal decomposition of the nanocomposites. Pack et al. [43] reported that CNT layers exhibited a good barrier effect to hinder the transport of volatile decomposed products on the thermal degradation as well as the slowdown the thermal decomposition of the nanocomposites. The CNT also acts as a reinforcing material and restricted the chain mobility of the polymer. Similar observation has also been reported where CNT layers exhibited a good barrier effect on the thermal degradation process, leading to the retardation of the weightloss rate of thermal degradation products as well as thermal insulation of polymer in the nanocomposites [44]. The $T_{1}$ and $T_{50}$ of the nanocomposites were little increased with the increasing amount of MWCNT, indicating that the MWCNTs were welldispersed in the PC matrix and well-interacted with the PC chains at low loading. Consequently, a small amount of MWCNT is sufficient to create the inflammable jammed network that retards transport of the decomposition products.

\subsubsection{DMA}

The dynamic mechanical behavior of pure $\mathrm{PC}$ and its nanocomposites, prepared at $350^{\circ} \mathrm{C}$, with different loading of MWCNTs is shown in Figure 7. From the figure, it can be seen that storage modulus of the pure PC was improved with incorporation of

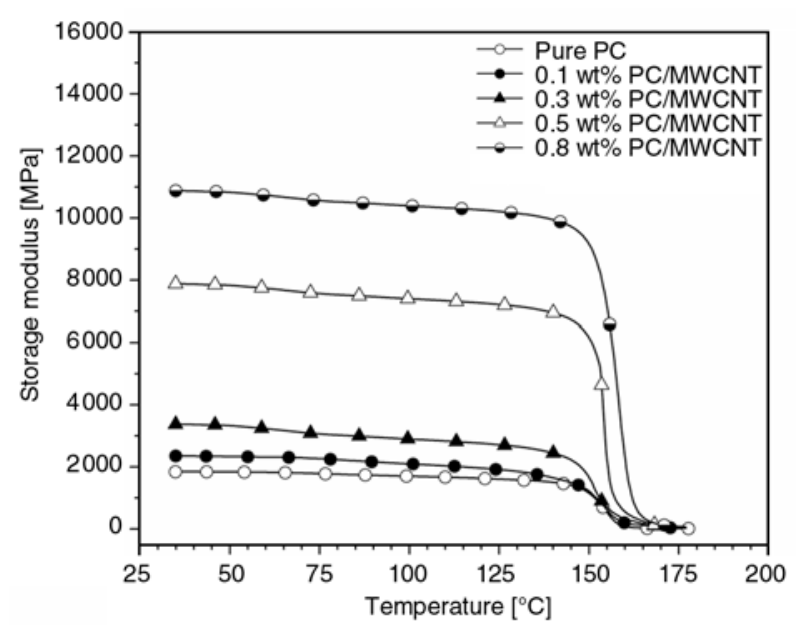

Figure 7. Plot of storage modulus vs. temperature for pure $\mathrm{PC}$, and PC/MWCNT nanocomposites containing $0.1 \mathrm{wt} \%$ MWCNT loading, $0.3 \mathrm{wt} \%$ MWCNT loading, $0.5 \mathrm{wt} \%$ MWCNT loading, $0.8 \mathrm{wt} \%$ MWCNT loading. All the nanocomposites were prepared at $350^{\circ} \mathrm{C}$.

MWCNT in the PC/MWCNT nanocomposites. The well and homogeneous distribution of MWCNT throughout the polymer matrix is mainly accountable for increasing the dynamic mechanical behavior of the nanocomposites. Individual dispersion of the MWCNTs throughout the polymer matrix leads to utmost interaction between polymer and MWCNTs even at low MWCNT loading. This interaction is well known as interfacial interaction which play major role to increase the storage modulus of the nanocomposites. Thus, storage modulus of the nanocomposites enhanced sharply compared to the pure polymer with the incorporation of MWCNT. The high aspect ratio and large surface area of the MWCNT make a strong interfacial interaction with the neighboring polymer chains and developed an interphase zone, even at low loading of MWCNT [45]. In addition, the incorporated MWCNT acts as reinforcing agent in the nanocomposites which enhanced surface compatibility among the host polymer and MWCNT.

Sternstein and Zhu [46] studied the visco-elastic properties of the nanocomposites materials and reported that nanometric dimension of the nanofillers in the nanocomposites contribute to the process of tether chain entanglement, which might have significant impact on this interaction zone. The tethered zones will be more significant with the addition of high aspect ratio nanofillers in the nanocomposites because of high surface area of the filler. Thus, a cross linking physical bond has been 
developed between the polymers and nanofillers in the nanocomposites that reduced polymer chain relaxation in the nanocomposites. This restricted the movement of the polymeric chains in the nanocomposites, leading to an increase in storage modulus of the nanocomposites.

The $\tan \delta$ curve for the pure PC and its nanocomposites, prepared at $350^{\circ} \mathrm{C}$, with different loading of MWCNT is shown in Figure 8. As observed, $\tan \delta$ peaks for pure PC appeared at $\sim 161^{\circ} \mathrm{C}$. In the nanocomposites, $\tan \delta$ peaks are shifted towards higher temperature compared to pure $\mathrm{PC}$, indicating that MWCNT acts as a shielding material in the nanocomposites. The Horowiyz-Metzger analysis [47] discussed that the incorporation of the CNTs into the matrix polymer could raise the activation energy for thermal decomposition and lead to the enhancement of the thermal stability of the nanocomposites, resulting from physical barriers effects of CNTs against thermal decomposition. As observed, the $\tan \delta$ significantly increases with an increase in MWCNT loading and the damping peak is sharply decreased with the addition of MWCNTs. This is indicating that the MWCNTs in the nanocomposites significantly obstructed and restricted chain segmental motion of the PC. However, $T_{\mathrm{g}}$ determined from the tan? peak increased slightly with increase

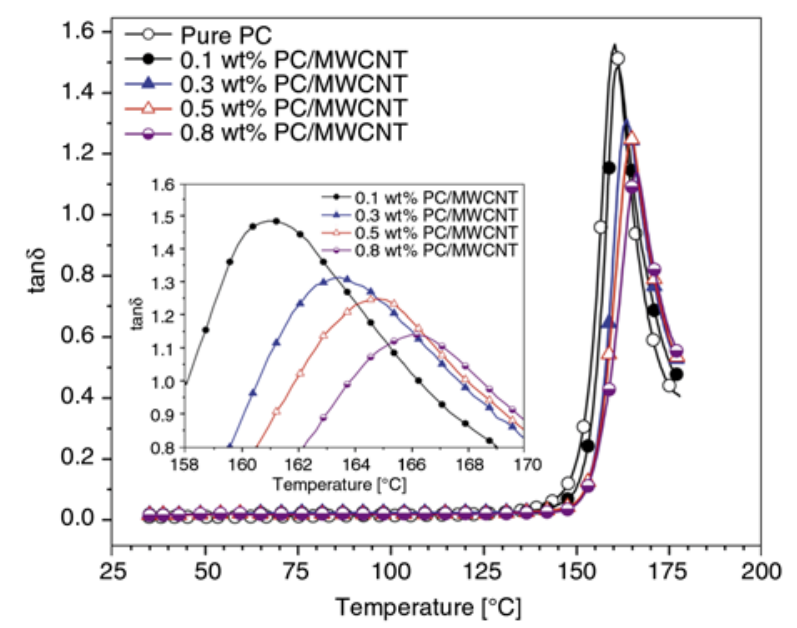

Figure 8. Plot of $\tan \delta$ vs. temperature for pure $\mathrm{PC}$, and $\mathrm{PC} /$ MWCNT nanocomposites containing $0.1 \mathrm{wt} \%$ MWCNT loading, $0.3 \mathrm{wt} \%$ MWCNT loading, $0.5 \mathrm{wt} \%$ MWCNT loading, $0.8 \mathrm{wt} \%$ MWCNT loading. The inset in Figure 8 represents the $\tan \delta$ vs. temperature for PC/MWCNT nanocomposites containing different MWCNT loading in the temperature region 158 to $170^{\circ} \mathrm{C}$. All the nanocomposites were prepared at $350^{\circ} \mathrm{C}$. in MWCNTs loading, from $\sim 161$ to $\sim 163^{\circ} \mathrm{C}, \sim 164$ and $\sim 166^{\circ} \mathrm{C}$ with the incorporation of $0.3,0.5$ and $0.8 \mathrm{wt} \%$ of MWCNTs loading, respectively.

\section{Conclusions}

In conclusion, an entire DC and AC electrical conductivity, dielectric permittivity of the PC/MWCNT nanocomposites, prepared at $350^{\circ} \mathrm{C}$, were carried out in broad frequency region at room temperature. The DC conductivity of the nanocomposites is measured at room temperature and very low percolation threshold $(0.11 \mathrm{wt} \%)$ was achieved. The frequency dependent dielectric permittivity behavior of the nanocomposites indicated that it can be applicable as well dielectric materials. This high electrical conductive PC/MWCNT nanocomposites was prepared by very easy and industrially feasible melt blending method at high temperature $\left(\sim 350^{\circ} \mathrm{C}\right)$ using internal mixer followed by compression molding without any purification or chemical modifications of the MWCNTs. The homogeneous and regular dispersion of MWCNTs throughout the matrix polymer is the reason behind the high electrical conductivity at very low concentration of MWCNTs. The linear variation in the plot of $\log \sigma_{\mathrm{DC}}$ versus $p^{-1 / 3}$ confirms the existence of tunneling conduction between MWCNTs. Thermal and storage modulus of the PC was also increased in the presence of small amount of MWCNTs.

\section{Acknowledgements}

We thank the Council of Scientific and Industrial Research (CSIR), New Delhi, India for financial support.

\section{References}

[1] Spitalsky Z., Tasis D., Papagelis K., Galiotis C.: Carbon nanotube-polymer composites: Chemistry, processing, mechanical and electrical properties. Progress in Polymer Science, 35, 357-401 (2010).

DOI: 10.1016/j.progpolymsci.2009.09.003

[2] Li C. Y., Li L., Cai W., Kodjie S. L., Tenneti K. K.: Nanohybrid shish-kebabs: Periodically functionalized carbon nanotubes. Advanced Materials, 17, 11981202 (2005).

DOI: $10.1002 / \mathrm{adma} .200401977$

[3] Yoshimura S. H., Khan S., Maruyama H., Nakayama Y., Takeyasu K.: Fluorescence labeling of carbon nanotubes and visualization of a nanotube-protein hybrid under fluorescence microscope. Biomacromolecules, 12, 1200-1204 (2011).

DOI: $\underline{10.1021 / \mathrm{bm} 101491 \mathrm{~s}}$ 
[4] Geng J., Zeng T.: Influence of single-walled carbon nanotubes induced crystallinity enhancement and morphology change on polymer photovoltaic devices. Journal of the American Chemical Society, 128, $16827-$ 16833 (2006).

DOI: $10.1021 /$ ja065035z

[5] Tang Y., Allen B. L., Kauffman D. R., Star A.: Electrocatalytic activity of nitrogen-doped carbon nanotube cups. Journal of the American Chemical Society, 131, 13200-13201 (2009).

DOI: $10.1021 /$ ja904595t

[6] Gajendran P., Saraswathi R.: Enhanced electrochemical growth and redox characteristics of poly $(o$-phenylenediamine) on a carbon nanotube modified glassy carbon electrode and its application in the electrocatalytic reduction of oxygen. Journal of Physical Chemistry C, 111, 11320-11328 (2007). DOI: $10.1021 / j p 071848 d$

[7] Vijayaraghavan A., Blatt S., Weissenberger D., OronCarl M., Hennrich F., Gerthsen D., Hahn H., Krupke R.: Ultra-large-scale directed assembly of single-walled carbon nanotube devices. Nano Letters, 7, 1556-1560 (2007).

DOI: $10.1021 / \mathrm{n} 10703727$

[8] Moniruzzaman M., Winey K. I.: Polymer nanocomposites containing carbon nanotubes. Macromolecules, 39, 5194-5205 (2006).

DOI: $10.1021 / \mathrm{ma} 060733 \mathrm{p}$

[9] Wu T-M., Chen E-C., Lin Y-W., Chiang M-F., Chang G-Y.: Preparation and characterization of melt-processed polycarbonate/multiwalled carbon nanotube composites. Polymer Engineering and Science, 48, 1369-1375 (2008).

DOI: $10.1002 /$ pen.21094

[10] Hornbostel B., Pötschke P., Kotz J., Roth S.: Singlewalled carbon nanotubes/polycarbonate composites: Basic electrical and mechanical properties. Physica Status Solidi B, 243, 3445-3451 (2006).

DOI: $10.1002 /$ pssb.200669199

[11] Sathpathy B. K., Weidisch R., Pötschke P., Janke A.: Tough-to-brittle transition in multiwalled carbon nanotube (MWNT)/polycarbonate nanocomposites. Composites Science and Technology, 67, 867-879 (2007). DOI: 10.1016/j.compscitech.2006.01.036

[12] Yoon H., Okamoto K., Umishita K., Yamaguchi M.: Development of conductive network of multiwalled carbon nanotubes in polycarbonate melt. Polymer Composites, 32, 97-102 (2011).

DOI: $10.1002 / \mathrm{pc} .21024$

[13] Linares A., Canalda J. C., Cagiao M. E., GarcíaGutiérrez M. C., Nogales A., Martín-Gullón I., Vera J., Ezquerra T. A.: Broad-band electrical conductivity of high density polyethylene nanocomposites with carbon nanoadditives: Multiwall carbon nanotubes and carbon nanofibers. Macromolecules, 41, 7090-7097 (2008).

DOI: $10.1021 / \mathrm{ma} 801410 \mathrm{j}$
[14] Newman D., Laredo E., Bello A., Grillo A., Feijoo J. L., Müller A. J.: Molecular mobilities in biodegradable poly(DL-lactide)/poly( $\varepsilon$-caprolactone) blends. Macromolecules, 42, 5219-5225 (2009).

DOI: $10.1021 / \mathrm{ma9007303}$

[15] Fisch R., Harris A. B.: Critical behavior of random resistor networks near the percolation threshold. Physical Review B, 18, 416-420 (1978).

DOI: 10.1103/PhysRevB.18.416

[16] Yu J., Lu K., Sourty E., Grossiord N., Koning C. E., Loos J.: Characterization of conductive multiwall carbon nanotube/polystyrene composites prepared by latex technology. Carbon, 45, 2897-2903 (2007).

DOI: $10.1016 /$ j.carbon.2007.10.005

[17] Ezquerra T. A., Kulescza M., Baltá-Calleja F. J.: Electrical transport in polyethylene-graphite composite materials. Synthetic Metals, 41, 915-920 (1991). DOI: 10.1016/0379-6779(91)91526-G

[18] Balberg I., Binenbaum N., Wagner N.: Percolation thresholds in the three-dimensional sticks system. Physical Review Letters, 52, 1465-1468 (1984).

DOI: $10.1103 /$ PhysRevLett.52.1465

[19] Grossiord N., Loos J., van Laake L., Maugey M., Zakri C., Koning C. E., Hart A. J.: High-conductivity polymer nanocomposites obtained by tailoring the characteristics of carbon nanotube fillers. Advanced Functional Materials, 18, 3226-3234 (2008). DOI: $10.1002 / \mathrm{adfm} .200800528$

[20] Ryvkina N., Tchmutin I., Vilčáková J., Pelíšková M., Sáha P.: The deformation behavior of conductivity in composites where charge carrier transport is by tunneling: Theoretical modeling and experimental results. Synthetic Metals, 148, 141-146 (2005). DOI: 10.1016/j.synthmet.2004.09.028

[21] Wang M., Li B., Wang J., Bai P.: Preparation and properties of polysiloxane grafting multi-walled carbon nanotubes/polycarbonate nanocomposites. Polymers for Advanced Technologies, 22, 1738-1746 (2011). DOI: $10.1002 /$ pat.1665

[22] Gefen Y., Aharony A., Alexander S.: Anomalous diffusion on percolating clusters. Physical Review Letters, 50, 77-80 (1983).

DOI: 10.1103/PhysRevLett.50.77

[23] Laredo E., Grimau M., Bello A., Wu D. F., Zhang Y. S., Lin D. P.: AC conductivity of selectively located carbon nanotubes in poly( $\varepsilon$-caprolactone)/polylactide blend nanocomposites. Biomacromolecules, 11, 13391347 (2010).

DOI: $10.1021 / \mathrm{bm} 100135 \mathrm{n}$

[24] Bello A., Laredo E., Marval J. R., Grimau M., Arnal M. L., Müller A. J.: Universality and percolation in biodegradable poly( $\varepsilon$-caprolactone)/multiwalled carbon nanotube nanocomposites from broad band alternating and direct current conductivity at various temperatures. Macromolecules, 44, 2819-2828 (2011). DOI: $10.1021 / \mathrm{ma1} 02598 \mathrm{~h}$ 
[25] Kilbride B. E., Coleman J. N., Fraysse J., Fournet P., Cadek M., Drury A., Hutzler S., Roth S., Blau W. J.: Experimental observation of scaling laws for alternating current and direct current conductivity in polymercarbon nanotube composite thin films. Journal of Applied Physics, 92, 4024-4030 (2002).

DOI: $10.1063 / 1.1506397$

[26] Balberg I.: Tunneling and nonuniversal conductivity in composite materials. Physical Review Letters, 59, 1305-1308 (1987).

DOI: $10.1103 /$ PhysRevLett.59.1305

[27] Sun X., Song M.: Highly conductive carbon nanotube/polymer nanocomposites achievable? Macromolecular Theory and Simulations, 18, 155-161 (2009). DOI: $10.1002 /$ mats.200800091

[28] Wu D., Zhang Y., Zhang M., Yu W.: Selective localization of multiwalled carbon nanotubes in poly( $\varepsilon$-caprolactone)/polylactide blend. Biomacromolecules, 10, 417-424 (2009).

DOI: $10.1021 / \mathrm{bm} 801183 \mathrm{f}$

[29] Dyre J. C.: The random free-energy barrier model for ac conduction in disordered solids. Journal of Applied Physics, 64, 2456-2468 (1988).

DOI: $10.1063 / 1.341681$

[30] Von Hippel A. R.: Dielectrics and waves. MIT Press, Cambridge (1996).

[31] Dyre J. C., Schrøder T. B.: Universality of ac conduction in disordered solids. Reviews of Modern Physics, 72, 873-892 (2000).

DOI: 10.1103/RevModPhys.72.873

[32] Straley J. P.: Critical exponents for the conductivity of random resistor lattices. Physical Review B, 15, 57335737 (1977).

DOI: $10.1103 /$ PhysRevB.15.5733

[33] Ahmad M. M., Yamada K., Okuda T.: Frequency dependent conductivity and dielectric studies on $\mathrm{RbSn}_{2} \mathrm{~F}_{5}$. Solid State Communications, 123, 185-189 (2002). DOI: 10.1016/S0038-1098(02)00300-9

[34] Lu H., Zhang X., Zhang H.: Influence of the relaxation of Maxwell-Wagner-Sillars polarization and dc conductivity on the dielectric behaviors of nylon 1010 . Journal of Applied Physics, 100, 054104/1-054104/7 (2006).

DOI: $10.1063 / 1.2336494$

[35] Neagu R. M., Neagu E., Bonanos N., Pisis P.: Electrical conductivity studies in nylon 11. Journal of Applied Physics, 88, 6669-6677 (2000).

DOI: $10.1063 / 1.1323752$

[36] Grimau M., Laredo E., Pérez Y. M. C., Bello A.: Study of dielectric relaxation modes in poly( $\varepsilon$-caprolactone): Molecular weight, water sorption, and merging effects. The Journal of Chemical Physics, 114, 6417-6425 (2001).

DOI: $\underline{10.1063 / 1.1355288}$
[37] Scher H., Lax M.: Stochastic transport in a disordered solid. I. Theory. Physical Review B, 7, 4491-4502 (1973).

DOI: 10.1103/PhysRevB.7.4491

[38] Barrau S., Demont P., Peigney A., Laurent C., Lacabanne C.: DC and AC conductivity of carbon nanotubes-polyepoxy composites. Macromolecules, 36, 5187-5194 (2003).

DOI: $10.1021 / \mathrm{ma} 021263 \mathrm{~b}$

[39] Schaefer D. W., Justice R. S.: How nano are nanocomposites? Macromolecules, 40, 8501-8517 (2007).

DOI: 10.1021/ma070356w

[40] Dissado L. A., Hill R. M.: Anomalous low-frequency dispersion. Near direct current conductivity in disordered low-dimensional materials. Journal of the Chemical Society, Faraday Transactions 2: Molecular and Chemical Physics, 80, 291-319 (1984).

DOI: 10.1039/F29848000291

[41] Lewis T. J.: Interfaces are the dominant feature of dielectrics at the nanometric level. IEEE Transactions on Dielectrics and Electrical Insulation, 11, 739-753 (2004). DOI: $10.1109 /$ TDEI.2004.1349779

[42] O'Konski C. T.: Electric properties of macromolecules. V. Theory of ionic polarization in polyelectrolytes. The Journal of Physical Chemistry, 64, 605619 (1960). DOI: $10.1021 / \mathrm{j} 100834 \mathrm{a} 023$

[43] Pack S., Kashiwagi T., Stemp D., Koo J., Si M., Sokolov J. C., Rafailovich M. H.: Segregation of carbon nanotubes/organoclays rendering polymer blends self-extinguishing. Macromolecules, 42, 6698-6709 (2009). DOI: $10.1021 / \mathrm{ma900966k}$

[44] Kuilla T., Bhadra S., Yao D., Kim N. H., Bose S., Lee J. H.: Recent advances in graphene based polymer composites. Progress in Polymer Science, 35, 1350 1375 (2011). DOI: $10.1016 / j . p r o g p o l y m s c i .2010 .07 .005$

[45] Orhan T., Isitman N. A., Hacaloglu J., Kaynak C.: Thermal degradation of organophosphorus flame-retardant poly(methyl methacrylate) nanocomposites containing nanoclay and carbon nanotubes. Polymer Degradation and Stability, 97, 273-280 (2012). DOI: $10.1016 /$ j.polymdegradstab.2011.12.020

[46] Sternstein S. S., Zhu A-J.: Reinforcement mechanism of nanofilled polymer melts as elucidated by nonlinear viscoelastic behavior. Macromolecules, 35, 7262-7273 (2002).

DOI: $10.1021 / \mathrm{ma} 020482 \mathrm{u}$

[47] Horowitz H. H., Metzger G.: A new analysis of thermogravimetric traces. Analytical Chemistry, 35, 14641468 (1963).

DOI: $10.1021 / \mathrm{ac} 60203 \mathrm{a} 013$ 